01

\title{
Синхронизация в турбулентном сферическом течении Куэтта под действием неравномерного вращения
}

\author{
(С) Д.Ю. Жиленко, О.Э. Кривоносова \\ Институт механики МгУ им. М.В. Ломоносова, \\ 119192 Москва, Россия \\ e-mail: jilenko@imec.msu.ru
}

Поступило в Редакцию 9 ноября 2018 г.

В окончательной редакции 9 ноября 2018 г.

Принято к публикации 22 января 2019 г.

\begin{abstract}
Представлены результаты численного исследования свойств турбулентных течений вязкой несжимаемой жидкости во вращающемся сферическом слое при наличии синхронизации. Синхронизация турбулентного течения происходит под действием периодического изменения скорости вращения внутренней сферы, скорость вращения внешней сферы постоянна. Проведено сравнение с экспериментом, и определен диапазон амплитуд модуляции, при котором синхронизация сопровождалась перемежаемостью типа „хаос-хаос“.
\end{abstract}

DOI: 10.21883/JTF.2019.07.47786.391-18

\section{Введение}

Синхронизация, как явление взаимодействия между осциллирующими объектами [1], встречается в физических, химических и биологических системах [2] а также во многих практических приложениях, например, при передаче информации [3]. Одним из ее наиболее распространенных видов является фазовая синхронизация, предполагающая захват фазы в системе внешним осциллятором [4]. Вблизи границы области синхронизации может наблюдаться перемежаемость $[5,6]$ в виде произвольного чередования во времени или/и в пространстве участков с различным динамическим поведением. Как синхронизация, так и связанная с ней перемежаемость наблюдаются и изучаются в природных системах, таких как турбулентные течения в атмосфере Земли [7-10]. В связи с этим получило распространение и лабораторное моделирование процессов синхронизации турбулентных течений внешними, периодическими во времени воздействиями: с учетом неизотермичности жидкости [11] и вращения [12]. Влияние на крупномасштабные процессы в атмосфере Земли оказывают в числе многих других факторов вращение и сферическая геометрия, которые являются определяющими в сферическом течении Куэтта - течении вязкой несжимаемой жидкости между двумя концентрически расположенными сферами, вращающимися вокруг общей оси. Результаты, полученные при исследовании сферического течения Куэтта в лабораторном эксперименте [13], показали возможность изменения локальных свойств турбулентности под действием периодической модуляции скорости вращения, по крайне мере в той области течения, где проводились измерения. Однако в перечисленных выше работах не рассматривался вопрос о зависимости интегральных свойствах турбулентных течений от параметров модуляции. Цель настоящей работы состоит в численном исследовании влияния синхронизации, вызываемой мо- дуляцией скорости вращения, на изменение локальных и интегральных свойств турбулентных течений, а также на появление перемежаемости.

\section{Численный метод и область исследования}

Для описания изотермического течения вязкой несжимаемой жидкости в сферическом слое используются уравнения Навье-Стокса и неразрывности

$\frac{\partial \mathbf{U}}{\partial t}=\mathbf{U} \times \operatorname{rot} \mathbf{U}-\operatorname{grad}\left(\frac{p}{\rho}+\frac{\mathbf{U}^{2}}{2}\right)-v \operatorname{rot} \operatorname{rot} \mathbf{U}, \operatorname{div} \mathbf{U}=0$.

Условия прилипания и не протекания на границах слоя, представленные в сферической системе координат с радиальным $(r)$, полярным $(\theta)$ и азимутальным $(\varphi)$ направлениями, имеют вид

$$
\begin{gathered}
u_{\varphi}\left(r=r_{k}\right)=\Omega_{k}(t) r_{k} \sin \theta, \quad u_{r}\left(r=r_{k}\right)=0, \\
u_{\theta}\left(r=r_{k}\right)=0, \quad k=1,2
\end{gathered}
$$

(1 - внутренняя сфера, 2 - внешняя). Здесь $\mathbf{U}, p, \rho-$ скорость, давление и плотность жидкости, $u_{\varphi}, u_{r}, u_{\theta}-$ соответственно азимутальная, радиальная и полярная компоненты скорости, $\Omega_{k}-$ угловая скорость вращения соответствующей сферы, $v$ - кинематическая вязкость жидкости в слое. В настоящей работе скорость вращения внешней сферы $\Omega_{2}$ оставалась постоянной, скорость вращения внутренней сферы изменялась периодически: $\Omega_{1}(t)=\Omega_{10}(1+A \sin (2 \pi g t+\varphi)), A, g$ - амплитуда и частота модуляции, $\Omega_{10}-$ средняя скорость вращения внутренней сферы. Использовались алгоритм [14] и основанные на нем программы численного решения $[14,15]$ с конечно-разностной схемой дискретизации уравнений Навье-Стокса по пространству и полунеявной схемой Рунге-Кутты для интегрирования по 
времени. Дискретизация по пространству проводилась с уменьшением размера ячеек вблизи границ (по $r$ ) и плоскости экватора (по $\theta$ ). Отношение максимального размера ячейки к минимальному изменялось от 2 до 4 , с общим количеством узлов до $1.15 \cdot 10^{6}$. Зависимость результатов расчетов от конфигурации расчетной сетки изучалась во многих работах (например, в [16]). С целью сравнения с имеющимися экспериментальными данными расчеты проводились при размерных параметрах, соответствующих условиям проведения экспериментов в [13]: $v=5 \cdot 10^{-5} \mathrm{~m}^{2} / \mathrm{s}, \quad r_{1}=0.075 \mathrm{~m}, \quad r_{2}=0.15 \mathrm{~m}$, $g=0.08 \mathrm{~Hz}, A=0-0.12$. В качестве исходного турбулентного течения, подвергающегося воздействию модуляции, выбрано изучавшееся ранее численно течение, формирующееся в случае встречного вращения сферических границ с числами Рейнольдса $\operatorname{Re}_{1}=\Omega_{10} r_{1}^{2} / \nu=460$ и $\mathrm{Re}_{2}=\Omega_{2} r_{2}^{2} / v=-900$ соответственно для внутренней и внешней сфер [16]. В ходе численного эксперимента проводилась запись зависимости величин скорости течения от времени в точках, расположенных в меридиональной плоскости течения на лучах с угловыми отклонениями от оси вращения $\theta=21.3^{\circ}$ (вблизи оси вращения), $\theta=52.9^{\circ}$ (в средних широтах) и $\theta=89.8^{\circ}$ (в плоскости экватора) и относительными расстояниями от внутренней сферы $R=0.135,0.247,0.359,0.484,0.61$, 0.7 и 0.8 , где $R=\left(r-r_{1}\right) /\left(r_{2}-r_{1}\right)$. Точка с координатами $R=0.7$ и $\theta=52.9^{\circ}$ соответствует положению точки измерения азимутальной компоненты скорости в эксперименте [13]. Расчеты проводились следующим образом: расчет исходного течения при стационарных граничных условиях продолжался не менее $3.0 \cdot 10^{3} \mathrm{~s}$, после чего величина амплитуды $A$ на частоте модуляции изменялась от нуля до выбранного значения. Далее расчет для каждого выбранного значения $A$ продолжался не менее $1.5 \cdot 10^{3} \mathrm{~s}$, что составляет не менее 120 периодов колебаний скорости вращения внутренней сферы.

\section{Результаты}

Быстрое вращение является типичным условием формирования двумерной турбулентности с обратным направлением каскада передачи энергии в инерционном интервале на больших масштабах и прямым каскадом переноса энстрофии на малых масштабах [17]. Обратному каскаду соответствует зависимость энергетического спектра $E(k)$ от волнового числа $k$ в виде $E(k) \sim k^{-5 / 3}$, прямому каскаду $-E(k) \sim k^{-3}$ [17]. Как показывают экспериментальные данные [18], если течение с трехмерной турбулентностью (с прямым каскадом передачи энергии и наклоном спектра $-5 / 3$ на инерционном интервале) подвергнуть воздействию даже достаточно медленного вращения, то возможен переход к двумерной турбулентности. Дальнейшее увеличение скорости вращения смещает обратный каскад в область меньших масштабов. Подобные этому переходы от трехмерной турбулентности к двумерной наблюдаются и в атмосфе-

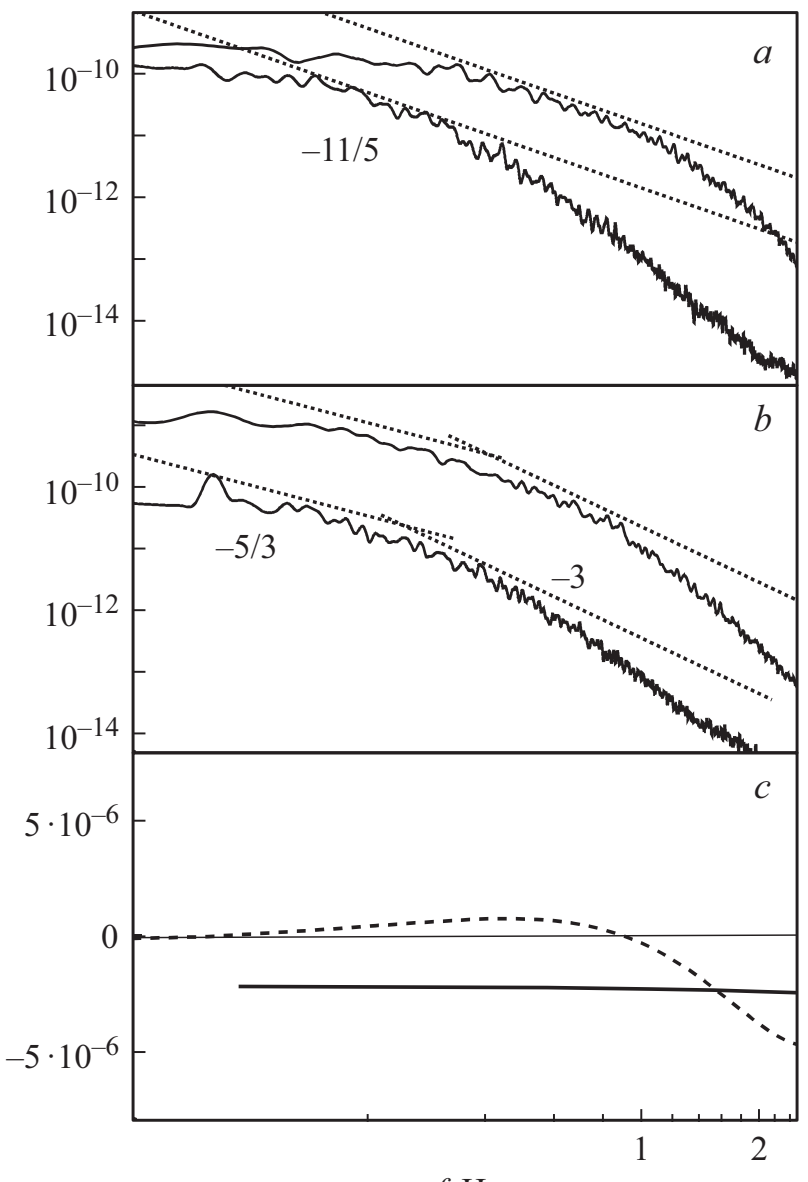

$f, \mathrm{~Hz}$

Рис. 1. Спектры $u_{\varphi}^{2}(a, b)$ и аппроксимация величины $D_{\mathrm{LLL}}$ полиномом четвертой степени $(c) . a-A=0, b-A=0.02$; верхние кривые - расчет, нижние - эксперимент [13], для удобства сравнения кривые сдвинуты по оси ординат. $c-$ $A=0$ сплошная линия, $A=0.03$ прерывистая линия, горизонтальная точечная прямая соответствует нулевому значению.

pe, например в пограничных слоях ураганов [19]. В турбулентных течениях с вращением и стратификацией численно показана возможность формирования трехмерной турбулентности с наклоном спектра $-11 / 5$ [20].

Изменения вида спектра турбулентности наблюдались и в экспериментах с изотермическими течениями во вращающемся сферическом слое. Так, в отсутствие модуляции в точке измерения, расположенной в средних широтах между экватором и полюсом, обнаружен спектр турбулентности с наклоном на инерционном интервале $-11 / 5$ [13], характерный для трехмерной турбулентности. С увеличением амплитуды модуляции в той же самой точке измерения, при достаточно высоких уровнях синхронизации между скоростью сферы и скоростью течения, наблюдался спектр с наклоном $-5 / 3$ на низких частотах и наклоном -3 на высоких частотах, соответствующий двумерной турбулентности. Выводы о типе турбулентности основываются не только на виде спектра, но и должны подтверждаться опреде- 


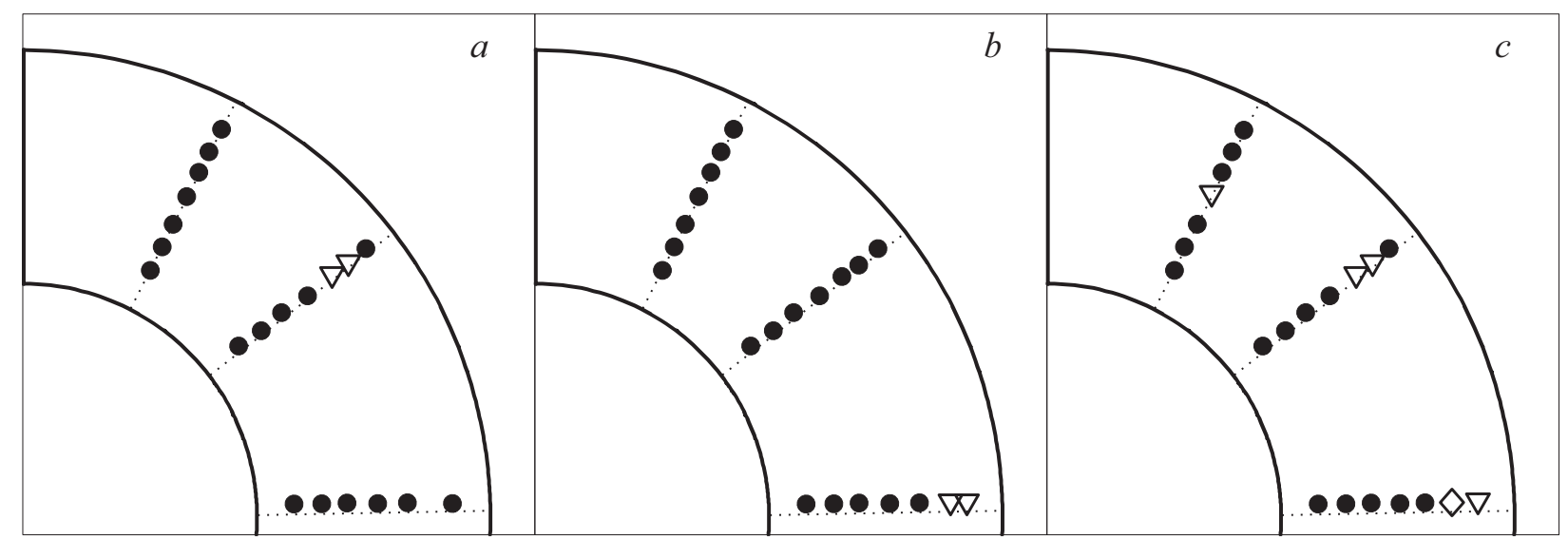

Рис. 2. Спектры $u_{\varphi}^{2}$ : • - соответствующие двумерной турбулентности; $\nabla-$ с наклоном $-11 / 5$, соответствующие трехмерной турбулентности; - с наклоном $-5 / 3$, соответствующие трехмерной турбулентности. $a-A=0, b-A=0.037, c-A=0.056$. В пропущенной точке постоянного угла наклона спектра не обнаружено.

лением направления каскада передачи энергии турбулентности, но в [13] такие данные не представлены. Направление каскада определяется по знаку продольной структурной функции скорости третьего порядка $\mathrm{D}_{\mathrm{LLL}}=\left\langle\left(u(l)-u\left(l^{1}\right)\right)^{3}\right\rangle[21], u-$ скорости течения в пространственно разнесенных точках $l$ и $l^{1}$, угловые скобки соответствуют осреднению по ансамблю реализаций. $\mathrm{D}_{\mathrm{LLL}}>0$ соответствует обратному каскаду, $\mathrm{D}_{\text {LLL }}<0$ соответствует прямому каскаду. На рис. 1 представлены результаты сравнения результатов экспериментов [13] с проведенным в настоящей работе расчетом спектров пульсаций азимутальной компоненты скорости течения $u_{\varphi}$, а также представлены расчетные величины $\mathrm{D}_{\text {LLL. }}$ Результаты приведены для точки, пространственное положение которой в слое соответствует условиям измерений в [13]. Видно, что как в эксперименте, так и в расчете при отсутствии модуляции (рис. $1, a$ ) в спектре наблюдается участок с постоянным углом наклона $-11 / 5$, а $D_{\text {LLL }}<0$ в рассматриваемом диапазоне частот (рис. 1,c), что, как уже отмечалось, соответствует трехмерной турбулентности с прямым каскадом передачи энергии. При ненулевой модуляции (рис. $1, b$ ) на меньших частотах наклон спектра близок к величине $-5 / 3$, на больших частотах наклон близок к -3 . Величина $\mathrm{D}_{\mathrm{LLL}}$ определялась таким же, как и в [22] способом, когда для вычисления использовалась зависимость $u_{\varphi}$ от азимутального угла $\varphi$ в течение 16 периодов вращения $(0<\varphi<32 \pi)$, а величина частоты (рис. $1, c)$ определялась как $f=\left\langle u_{\varphi}\right\rangle / l$, где $0<l<32 \pi r$, а $\left\langle u_{\varphi}\right\rangle-$ средняя скорость. Представленные на рис. 1 данные не достаточны для определения пространственного распределения различных видов турбулентности в сферическом слое. Ранее в [22] исследовался ламинарнотурбулентный переход под действием модуляции скорости вращения, и показана возможность формирования как двумерной, так и трехмерной турбулентности в различных областях течения, причем уровень синхрониза- ции для двумерной турбулентности оказался выше, чем для трехмерной. На рис. 2 показано пространственное распределение различных видов спектров турбулентности в меридиональной плоскости течения. Результаты расчетов показали, что при стационарных скоростях вращения $(A=0)$ обнаруженная ранее в экспериментах [13] трехмерная турбулентность, спектр которой содержит участок с постоянным наклоном $-11 / 5$, занимает только ограниченную область течения в средних широтах (рис. 2, a) - две точки вблизи внешней сферы $(R=0.61$ и 0.7). Во всей остальной области течения наблюдаются спектры, вид которых соответствует двумерной турбулентности. С увеличением амплитуды модуляции $(0.007<A<0.03)$ в средних широтах, так же как в эксперименте [13], вид спектров соответствует спектрам двумерной турбулентности. Начиная с $A=0.037$ (рис. $2, b$ ), на экваторе вблизи внешней сферы опять появляется спектр с наклоном $-11 / 5$. При дальнейшем увеличении $A$ такой спектр начинает распространяться на средние и высокие широты (рис. 2,c), сокращая, таким образом, занимаемую двумерной турбулентностью область течения. Необходимо отметить, что наклон спектра полной кинетической энергии течения близок к величине -5 , что согласуется с многочисленными результатами, приведенными в обзоре [17], причем наклон спектра не меняется с изменением амплитуды модуляции.

Модуляция скорости вращения может приводить к генерации среднего течения, вследствие чего могут изменяться как профили азимутальной компоненты скорости $u_{\varphi}$, так и величины ее среднеквадратичных отклонений

$$
\operatorname{rms} u_{\varphi}=\sqrt{\frac{1}{N_{t}} \sum_{n=1}^{N_{i}}\left(u_{\varphi}(n, r)-\overline{u_{\varphi}(r)}\right)^{2}} .
$$

Величины $u_{\varphi}$ и $\operatorname{rms} u_{\varphi}$, нормированные на эти же величины, но в отсутствие модуляции, $u_{n}=u_{\varphi} / u_{\varphi(A=0)}$ и 


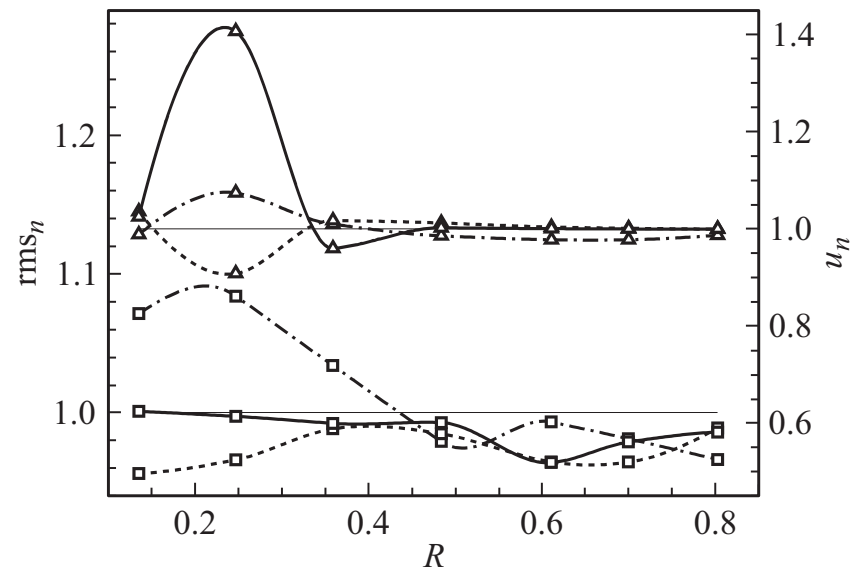

Рис. 3. Зависимость нормированных величин азимутальной скорости $u_{n}$, (треугольные символы) и ее среднеквадратичных отклонений $\mathrm{rms}_{n}$, (прямоугольные символы) от нормированного расстояния между сферами $R$ для трех значений меридионального угла в случае $A=0.007$. Прерывистые линии с точками $-\theta=21.3^{\circ}$, прерывистые линии $-\theta=52.9^{\circ}$, сплошные линии $-\theta=89.8^{\circ}$. Горизонтальные линии соответствуют величинам, равным единице.

$\mathrm{rms}_{n}=\mathrm{rms} u_{\varphi} / \mathrm{rms} u_{\varphi(A=0)}$, приведены на рис. 3. При малых амплитудах модуляции $A=0.007$ наиболее сильное изменение величин $u_{n}$ наблюдается в области влияния внутренней сферы, вблизи $R=0.247$ (рис. 3, верхняя часть), в остальных областях течения отношение скоростей близко к единице. Величины $\mathrm{rms}_{n}$ повышаются только вблизи оси вращения $\left(\theta=21.3^{\circ}\right)$ при $R<0.48$, тогда как на средних широтах и вблизи экватора во всем диапазоне изменения $R$ наблюдается небольшое снижение (до 5\%) этой величины (рис. 3). Таким образом, при малых амплитудах модуляции во всем течении за исключением приосевой области возможно снижение уровня турбулентных пульсаций скорости по сравнению со случаем $A=0$, что не противоречит результатам экспериментов [13].

Как интегральные параметры течения, так и скорости в каждой точке течения неоднородны во времени (рис. 4). Наблюдается произвольное чередование во времени участков с сильной турбулентностью, где синхронизация не заметна, и участков со слабой турбулентностью, на которых скорость внутренней сферы и скорость течения изменяются синхронно. Таким образом, в присутствии модуляции наблюдается перемежаемость типа „хаос-хаос“. Ранее перемежаемость „хаос-хаос“ во вращающихся сферических слоях определяли по произвольному чередованию участков с различной амплитудой турбулентных пульсаций (например, в [23]). В настоящей работе, так же как и в [24], показано, что участки с различным уровнем турбулентности отличаются в основном видом сигнала скорости. Участки со слабой турбулентностью, как хорошо заметно на рис. 4, не соответствуют по частоте сигналу скорости вращения внутренней сферы. Это может быть связано с тем, что так же как и при стационарных граничных условиях [16], при модуляции скорости вращения границы в турбулентном течении сохраняются фрагменты пространственной структуры, характерной для предшествующего переходу к турбулентности течения. Это течение при рассматриваемых в настоящей работе и в [16] средних числах Рейнольдса периодическое как по времени, так и по пространству (в азимутальном направлении).

Модуляция скорости вращения внутренней сферы представляет собой внешнюю по отношению к течению периодическую вынуждающую силу, которая может приводить к синхронизации между изменяющейся скоростью вращения внутренней сферы и всеми параметрами течения. Синхронизация представляет собой захват фазы и/или частоты. В настоящей работе уровень синхронизации определяется по временному поведению разности мгновенных фаз вынуждающей силы и величин, характеризующих рассматриваемое течение. Для вычисления мгновенных фаз скорости и/или других

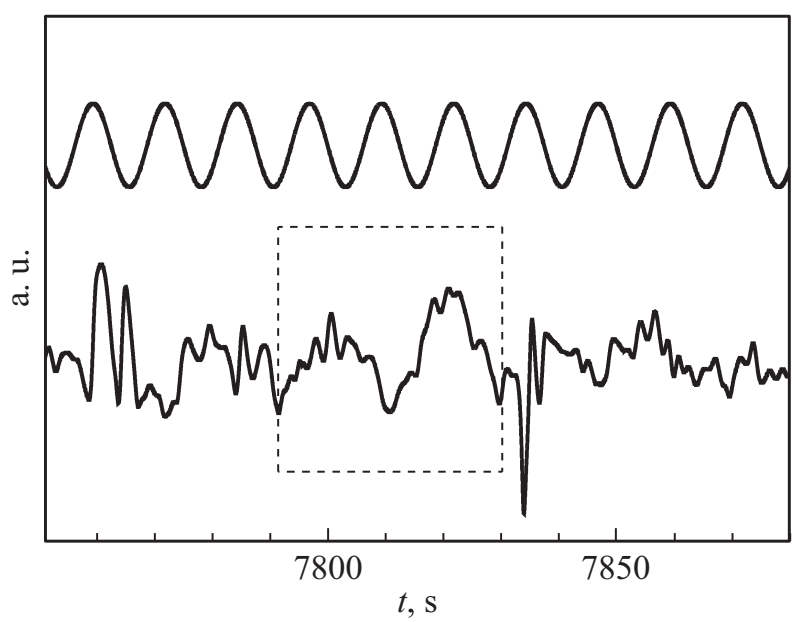

Рис. 4. Зависимость от времени $\Omega_{1}$ (сверху) и $u_{\varphi}$ в точке $\theta=21.3^{\circ}, R=0.484$ (внизу) при $A=0.007$. Прерывистой линией выделен фрагмент сигнала со слабой турбулентностью.

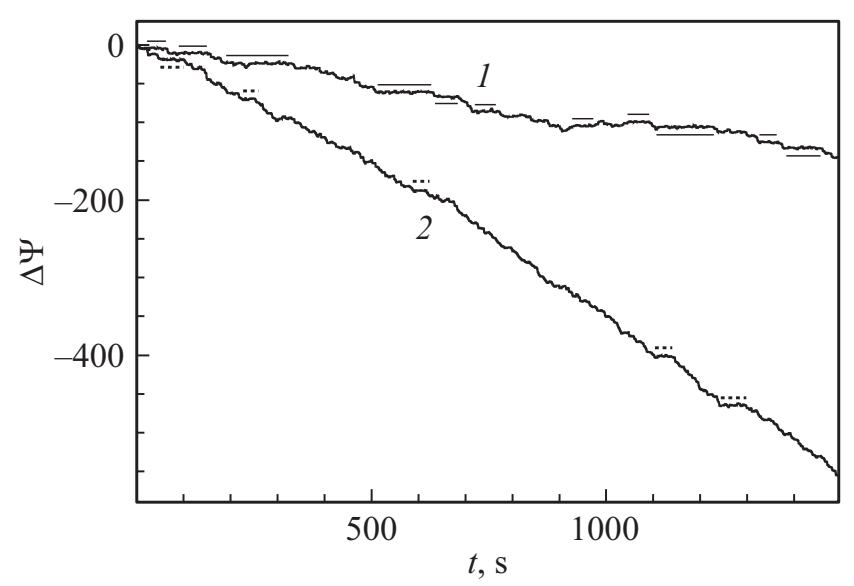

Рис. 5. Начальный фрагмент зависимости от времени величин разности фаз $\Delta \Psi$ между $\Omega_{1}$ и $E_{\varphi}(1)$ и между $\Omega_{1}$ и $E_{\psi}(2)$ при $A=0.0245$. 


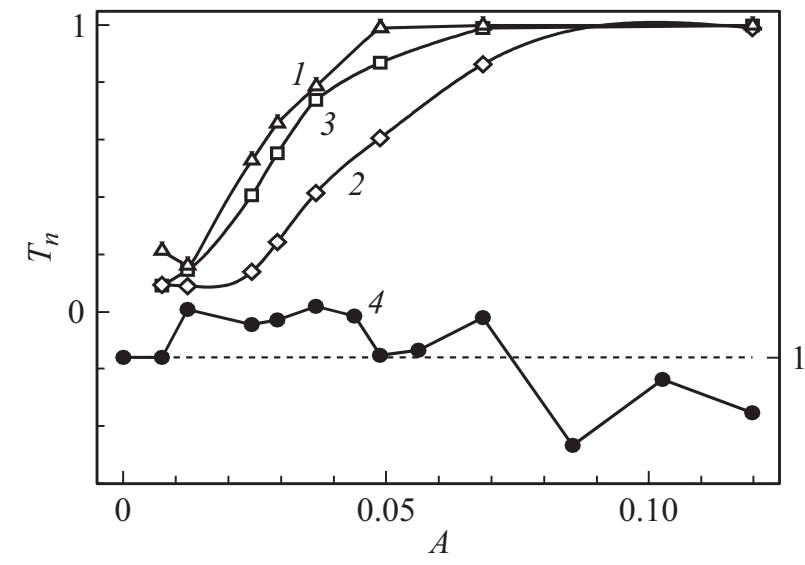

Рис. 6. Относительная продолжительность участков сильной синхронизации $T_{n}\left(E_{\varphi}\right)-1, T_{n}\left(E_{\psi}\right)-2$ и $T_{n}\left(M_{o}\right)-3$. Отношение $\left(E_{\varphi} / E_{\psi}\right) /\left(E_{\varphi} / E_{\psi}\right)_{A=0}-4$ (ось справа).

величин применяется метод [1,2], который ранее неоднократно использовался как в ламинарных, так и в турбулентных течениях (например, в $[12,13,22]$ ). Согласно этому методу, значение мгновенной фазы $\Psi(t)$ сигнала $x(t)$ определяется как $\Psi(t)=\operatorname{arctg}(y(t) / x(t)), y(t)-$ ортогональное дополнение к $x(t)$, которое вычисляется как преобразование Гильберта ряда $x(t)$. На рис. 5 представлены в зависимости от времени разности мгновенных фаз между скоростью вращения внутренней сферы $\Omega_{1}(t)$ и составляющими кинетической энергии течения - азимутальной $E_{\varphi}$ и меридиональной $E_{\psi}$ Эти составляющие определяются интегрированием по всему объему сферического слоя соответственно азимутальной $u_{\varphi}$ и меридиональных $u_{r}$ и $u_{\theta}$ компонент скорости течения $E_{\varphi}=\int u_{\varphi}^{2}, E_{\psi}=\int\left(u_{r}^{2}+u_{\theta}^{2}\right)$. Видно, что в представленных зависимостях есть участки, где в среднем разница фаз постоянна во времени (показаны горизонтальными линиями), это участки с сильной фазовой синхронизацией. Те участки, где фаза в среднем линейно изменяется со временем, соответствуют слабой фазовой синхронизации. Из рис. 5 видно, что наклон кривой 2 больше, а количество горизонтальных участков значительно меньше по сравнению с кривой 1. Это означает, что уровень синхронизации $E_{\varphi}$ (кривая 1 ) выше, чем уровень синхронизации $E_{\psi}$ (кривая 2 ). А именно модуляция скорости вращения границы воздействует, прежде всего на изменение именно азимутальной компоненты течения $u_{\varphi}$. Суммарная продолжительность участков с сильной синхронизацией (слабой турбулентностью), нормированная на общее время выборки, в зависимости от амплитуды модуляции $A$ для $E_{\varphi}\left(T_{n}\left(E_{\varphi}\right)\right), E_{\psi}\left(T_{n}\left(E_{\psi}\right)\right)$ и момента сил трения на внешней сфере

$$
M_{o}=v r_{2}^{3} \int_{0}^{2 \pi} \int_{0}^{2 \pi}\left(\frac{\partial v_{\varphi}}{\partial r}-\frac{v_{\varphi}}{r}\right) \sin ^{2} \theta d \theta d \varphi T_{n}\left(M_{o}\right)
$$

представлена на рис. 6. На оси ординат (слева) 0 соответствует отсутствию участков с сильной син- хронизацией, 1 - полной синхронизации. Видно, что кривые 1-3 располагаются выше нуля, что означает, что даже при самых малых величинах амплитуды модуляции $(A=0.007)$ в зависимостях интегральных параметров течения от времени присутствуют участки с сильной синхронизацией. Первой по мере возрастания $A$ достигает полной синхронизации $E_{\varphi}$ (кривая 1$)$, затем $M_{o}$ (кривая 3), и последней из представленных интегральных параметров $E_{\psi}$ (кривая 2). Также на рис. 6 представлено отношение составляющих кинетической энергии течения, нормированное на эту же величину в отсутствие модуляции $\left(E_{\varphi} / E_{\psi}\right) /\left(E_{\varphi} / E_{\psi}\right)_{A=0}$ (кривая 4). Следует отметить, что относительное изменение величины $E_{\psi}$ больше, чем относительное изменение величины $E_{\varphi}$. При $A>0.007$ величина $\left(E_{\varphi} / E_{\psi}\right) /\left(E_{\varphi} / E_{\psi}\right)_{A=0}$ начинает отличаться от значений, соответствующих стационарным граничным условиям. На участке $0.007<A<0.05$ величина $\left(E_{\varphi} / E_{\psi}\right) /\left(E_{\varphi} / E_{\psi}\right)_{A=0}$ становится больше единицы. И в этом же диапазоне амплитуд наблюдается перемежаемость „хаос-хаос“. Причем на верхней границе этого диапазона $(A=0.05)$ достигается полная синхронизация величины $E_{\varphi}$. При $0.05<A<0.056$ наблюдается кратковременное приближение $E_{\varphi}$ и $E_{\psi}$ к своим стационарным значениям. При амплитуде $A>0.07$ величина $\left(E_{\varphi} / E_{\psi}\right) /\left(E_{\varphi} / E_{\psi}\right)_{A=0}$ начинает уменьшаться и по мере дальнейшего увеличения $A$ становится меньше единицы. Все вышесказанное означает, что при неполной синхронизации хотя бы одной из составляющих кинетической энергии, когда наблюдается перемежаемость „хаос-хаос“, отношение $\left(E_{\varphi} / E_{\psi}\right)$ превышает свои стационарные значения. С увеличением амплитуды при достижении полной синхронизации всеми составляющими отношение $\left(E_{\varphi} / E_{\psi}\right)$ становится меньше своих стационарных значений. Это объясняется тем, что после достижения полной синхронизации $E_{\psi}$ под действием модуляции растет быстрее, чем $E_{\varphi}$. Таким образом, соотношение между азимутальной и меридиональной составляющими кинетической энергии течения определяется степенью синхронизации каждой из них с периодически изменяющейся скоростью вращения внутренней сферы.

\section{Заключение}

Численно изучена зависимость свойств турбулентных течений во вращающемся сферическом слое от амплитуды периодического изменения скорости вращения внутренней сферы. Впервые подтверждена показанная ранее в эксперименте возможность формирования в ограниченных областях изотермических течений вязкой несжимаемой жидкости спектра трехмерной турбулентности с постоянным наклоном $-11 / 5$ как в отсутствие модуляции, так и при ее наличии. Установлено, что с увеличением амплитуды модуляции занимаемая двумерной турбулентностью область течения может сокращаться. 
Показано, что в диапазоне амплитуд модуляции $0.012<A<0.069$ синхронизация между изменяющейся скоростью сферы и течением ведет к формированию перемежаемости типа „хаос-хаос“. Такая перемежаемость представляет собой произвольное чередование во времени участков со слабой турбулентностью (с высоким уровнем синхронизацией) и сильной турбулентностью (с низким уровнем синхронизации).

Установлена корреляция как локальных, так и осредненных свойств течений с границами области существования перемежаемости „хаос-хаос“. Внутри этих границ обнаружено скачкообразное изменение соотношения между азимутальной и меридиональной составляющими кинетической энергии течения, поскольку полная синхронизация азимутальной составляющей происходит при меньших значениях $A$, чем меридиональной. При малых значениях $A$ показана возможность уменьшения уровня турбулентных пульсаций скорости по сравнению с той же величиной в отсутствие модуляции, что соответствует имеющимся экспериментальным результатам.

\section{Финансирование работы}

Работа выполнена при частичной финансовой поддержке РФФИ, проекты 19-05-00028 и 18-08-00074.

\section{Список литературы}

[1] Pikovsky A., Rosenblum M., Kurths J. Synchronization: A Universal Concept in Nonlinear Sciences. NY:: Cambridge University Press, 2001.

[2] Boccaletti S., Kurths J., Osipov G., Valladeres D., Zhou C. // Phys. Rep. 2002. Vol. 366. P. 1-101.

[3] Короновский А.А, Москаленко О.И., Павлов А.С., Фролов Н.С., Храмов А.Е. // ЖТФ. 2014. Вып. 5. С. 1-8. [Koronovskii A.A., Moskalenko O.I., Pavlov A.S., Frolov N.S., Hramov A.E. // Technic. Physics. 2014. Vol. 59. 5. P. 629636.]

[4] Rosenblum M., Pikovsky A., Kurths J. // Phys. Rev. Lett. 1996. Vol. 76. P. 1804-1807.

[5] Rosenblum M., Pikovsky A., Kurths J. // Phys. Rev. Lett. 1997. Vol. 78. P. 4193-4196.

[6] Boccaletti S., Valladares D. // Phys. Rev. E. 2000. Vol. 62. P. 7497-7500.

[7] Read P., Castrejon-Pita A. // Q.J. R. Meteorol. Soc. 2012. Vol. 138. P. 1338-1349.

[8] Кулямин Д.В., Дымников В.П. // Изв. РАН. ФАО. 2010. T. 46. № 4. C. 467-486. [Kulyamin D.V., Dymnikov V.P. // Izvestia. Atmospheric and Oceanic Physics. Vol. 46. N 4. P. 432-450.]

[9] Курбацкий А.Ф., Курбачкая Л.И. // ПМТФ. 2013. Т. 54. № 4. C. 55-67. [Kurbatskii A.F., Kurbatskaya L.I. // J. Appl. Mech. Tech. Phys. 2013. Vol. 54. 4. P. 561-571.]

[10] Kit E., Hocut C., Liberzon D., Fernando H. // J. Fluid. Mech. 2017. Vol. 833. P. 745-772.

[11] Maza D., Vallone A., Mancini H., Bocaletti S. // Phys. Rev. Lett. 2000. Vol. 85. P. 5567-5570.

[12] Read P., Morice-Atkinson X., Allen E., Castrejon-Pita A. // Chaos. 2017. Vol. 27. P. 127001.
[13] Жиленко Д.Ю., Кривоносова О.Э. // Письма в ЖТФ. 2017. T. 43. Вып. 10. C. 87-94. [Zhilenko D.Y., Krivonosova O.E. // Tech. Phys. Lett. 2017. Vol. 43. 5. P. 493-495.]

[14] Nikitin N. // J. Comp. Phys. 2006. Vol. 217. P. 759-781.

[15] Кривоносова О.Э. // Дисс. к.ф.-м.н. М.: МГУ. 2007.

[16] Жиленко Д.Ю., Кривоносова О.Э. // ЖТФ. 2010. Т. 80. Вып. 4. C. 16-23. [Zhilenko D.Y., Krivonosova O.E. // Technic. Physics. 2010. Vol. 55. N 4. P. 449-456.]

[17] Данилов С.Д., Гурарий Д. // УФН. 2000. Т. 170. № 9. C. 922-968. [Danilov S.D., Gurarie D. // Uspekhi Fizicheskikh Nauk. 2000. Vol. 170. N 9. P. 921-968.]

[18] Campagne A., Gallet B., Moisy F., Cortet P. // Phys. Fluid. 2014. Vol. 26. P. 125112.

[19] Byrne D., Zhang J. // Geophys. Res. Lett. 2013. Vol. 40. P. 1439-1442.

[20] Rosenberg D., Pouquet A., Marino R., Mininni P. // Phys. Fluid. 2015. Vol. 27. P. 055105.

[21] Frisch U. Turbulence: The legacy of A.N. Kolmogorov. Cambridge: Cambridge University Press, 1995.

[22] Жиленко Д.Ю., Кривоносова О.Э. // Письма в ЖЭТФ. 2015. T. 101. № 8. C. 583-588. [Zhilenko D., Krivonosova O. // JETP Lett. 2015. Vol. 101. N 8. P. 527-532.]

[23] Raynaud R., Dormy E. // Phys. Rev. E. 2013. Vol. 87. P. 033011.

[24] Жиленко Д.Ю., Кривоносова О.Э. // Докл. РАН. 2014. T. 454. № 3. C. 278-281. [Zhilenko D., Krivonosova O. // Dokl. Phys. 2014. 59. P. 45-48]. 